\title{
Limitation of motion
}

\author{
Nikhil A. Khomane \\ M.S.S. High School, Chinchwad, Pune-17(India)
}

\begin{abstract}
In this paper, I describe the actual limit of motion i.e. nothing can get complete stabilization nor can cross the speed of light. I use here two separate methods or formulas to prove it.
\end{abstract}

\section{Introduction:}

Here I use both Newtonian and Relativistic methods to prove. We always think that the stone or any other thing which cannot move by its own so we think that that stone is stable. But this is impossible cause the stone is actually moves with earth and earth moves around the sun and also the sun revolves around the galaxy with great speed. In this way nothing is stable. All of these concepts are relates with relativity.

The main effort is that to examine whether any matter in the universe is 'stable' or 'unstable'. And finding its results' effects on 'time'.

This idea also can little support for making 'UNIFIED THEORY OF EVERYTHING'... For everything in the universe, we can divide matter into two groups basically and that are 'stable matter' or 'unstable matter'...

1.Here by using Newton's formula "F=ma" we are going to prove that in the universe there is no any matter which is stable and if it exist then it is massless...

we know

$$
\mathbf{F}=\mathbf{m a}
$$

which is actually, $\mathbf{F}=\mathbf{m}(\mathbf{v}-\mathbf{u}) / \mathbf{t}$ therefore

$$
\mathbf{F}=(\mathbf{m v}-\mathbf{m u}) / \mathbf{t}
$$

For stable matter, the initial and final velocities become zero cause of its stableness.

Therefore according formula of Force,

$$
\begin{aligned}
& \mathrm{F}=(\mathbf{m . 0}-\mathrm{m} .0) / \mathrm{t} \\
& \mathrm{F}=(\mathbf{0 - 0}) / \mathrm{t}
\end{aligned}
$$

Therefore,

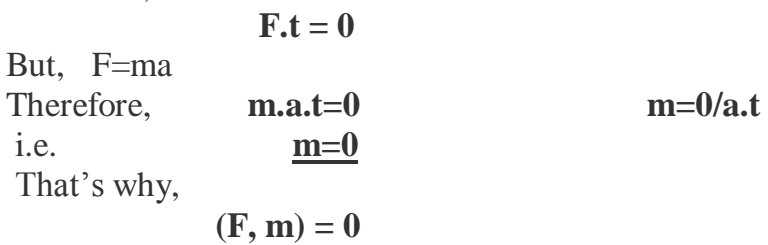

Therefore if matter is stable then it has no MASS and FORCE (to exert) ...and it will disappear and according to this we can say there is no any stable matter in the universe so everything is moving or in motion.....

'0' mass is also a possibility toward 'invisibility'...

According to this we also can conclude that nothing get complete stabilization or if this possible then it will nothing...

2. Here is also one thing that nothing can catch the speed of light.

If it will then it also will give same results that we got about stability.

For that, we know,

$$
\mathbf{E}=\mathbf{m c}^{2}
$$


Which is actually,

$$
E=\frac{m_{0} c^{2}}{\sqrt{1-v^{2} / c^{2}}}
$$

If object or matter catches the speed of light, then $v^{2}$ changes to $c^{2}$

Therefore,

$$
\begin{aligned}
E & =\frac{\mathrm{m}_{0} \mathrm{c}^{2}}{\sqrt{1-\mathrm{c}^{2} / \mathrm{c}^{2}}} \\
\mathbf{E} & =\frac{\mathrm{m}_{0} \mathrm{c}^{2}}{\sqrt{1-1}} \\
\mathbf{E} & =\frac{\mathrm{m}_{0} \mathrm{c}^{2}}{\sqrt{\mathbf{0}}} \\
\mathbf{E} & =\frac{\mathrm{m}_{0} \mathrm{c}^{2}}{\mathbf{0}} \\
\text { Therefore } \mathbf{E} \mathbf{0} & =\mathrm{m}_{0} \mathrm{c}^{2} \\
\mathbf{0} & =\mathrm{m}_{0} \mathrm{c}^{2}
\end{aligned}
$$

Finally we get $\mathbf{m}_{0}=\mathbf{0}$

So again we get our same result that object will massless and cause disappear

\section{Effects On Time:}

1. As we know by relativity about proper time and relativistic time, I am going to apply both the conditions that we applied earlier

We know, $\quad \frac{\mathbf{t}}{\mathbf{t}^{\prime}}=\frac{1}{\sqrt{1-\frac{v^{2}}{c^{2}}}}$

Again for stable object, velocity (v) is 0

$$
\begin{aligned}
& \frac{\mathrm{t}}{\mathrm{t}^{\prime}}=\frac{1}{\sqrt{1-\frac{0}{\mathrm{c}^{2}}}} \\
& \frac{\mathrm{t}}{\mathrm{t}^{\prime}}=\frac{1}{\sqrt{1-0}} \\
& \frac{\mathrm{t}}{\mathrm{t}^{\prime}}=\frac{1}{\sqrt{1}} \\
& \frac{\mathrm{t}}{\mathrm{t}^{\prime}}=\frac{1}{1}
\end{aligned}
$$

So we get that,

$$
\text { Proper time }(\mathrm{t})=\text { relativistic time }\left(\mathrm{t}^{\prime}\right)
$$

I.e. at this condition the object suffers from proper time as Einstein told that nothing can suffer from proper time inversely, as we got earlier that nothing can complete stable, so according to this we conclude that there is no anything or object which can suffer from proper time

So, via this, may be the life of any two or more same aged objects or living and non-living things is same, but still we can't recognize it cause of each or everyone's separate motion or speed in life. i.e. if we are considering here two healthy and same aged people, one's life is full of motion, hard work i.e. physical work and other one's is very sedentary and motion less,

Via formation, the person who do higher motion lives longer than the person who lives sedentary life i.e. second person die before first person as they both are fit, healthy and of same age.

2. Now applying our second condition i.e. the object is moving with speed of light

$$
\text { So again, } \quad \begin{aligned}
\frac{\mathrm{t}}{\mathrm{t}^{\prime}} & =\frac{1}{\sqrt{1-\frac{\mathrm{v}^{2}}{\mathrm{c}^{2}}}} \\
\frac{\mathrm{t}}{\mathrm{t}^{\prime}} & =\frac{1}{\sqrt{1-\frac{\mathrm{c}^{2}}{\mathrm{c}^{2}}}} \\
\frac{\mathrm{t}}{\mathrm{t}^{\prime}} & =\frac{1}{\sqrt{1-1}}
\end{aligned}
$$




$$
\begin{aligned}
\frac{\mathrm{t}}{\mathrm{t}^{\prime}} & =\frac{1}{\sqrt{0}} \\
\frac{\mathrm{t}}{\mathrm{t}^{\prime}} & =\frac{1}{0}
\end{aligned}
$$

Now we get,

$$
\text { Relativistic time }=0
$$

I.e. at speed of light time completely stop

But, as Einstein told that at speed of light, time stops completely as we showed.

As "as we move faster, time bits slower" and also according to him "if we cross the speed of light, the time for us or that object become negative."

I.e. it will go to 'past'

But as I told earlier that nothing can cross the speed of light, so going into past is impossible by this method.

\section{Final Conclusion}

No matter in the world can become complete stable or motion less or catch the speed limit i.e. speed of light so every speed should be more than ' $0 \mathrm{~m} / \mathrm{sec}$ ' or less than 'Speed of light'....

According to this I conclude that 'For Everything Stabilization is Nothing.

Relativity the general and special theory.

\section{Reference}

GLOSSARY: m-relativistic mass

$\mathrm{m}_{0}$-initial mass

F-force

t-proper time

t'-relativistic time

u-initial velocity

v-final velocity

c-speed of light

a-acceleration

\section{Nikhil A. Khomane}

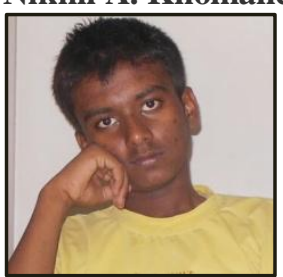

